\title{
Design and Application of Experimental Teaching Assistant Platform for Principle of Computer Composition
}

\author{
Lei Wei ${ }^{1, a}$, Qiuyun Zhao ${ }^{1, b}$ \\ ${ }^{1}$ School of Computer, Chengdu University of Information Technology, Chengdu, China \\ aweile@cuit.edu.cn, bzqyuen@cuit.edu.cn \\ * Lei Wei
}

Keywords: Principle of Computer Composition; Auxiliary teaching; Virtual experiment; Component.

\begin{abstract}
This paper discusses problems existing in the experimental teaching of Principle of Computer Composition. In order to strengthen the teaching objective of the course and improve students' practical ability and innovative thinking, an experimental teaching assistant platform is designed and developed based on MVC. The platform uses the SSM framework. The platform realizes functions of the resource sharing, student management, virtual experiment management, simulation program management, performance management, Q\&A and system management. And through the component technology, the platform has good expansibility. The application shows that the platform plays a good role in strengthening the communication between teachers and students, promoting the achievement of teaching objectives and cultivating students' abilities.
\end{abstract}

\section{Introduction}

Principle of Computer Composition is a major compulsory course in the computer science major. It plays an important role in the course system of hardware category and cultivating students' ability to analyze, develop, use and design computer hardware system [1]. In order to achieve the intended teaching objectives, the experimental teaching links are specially designed in the course. And through various means to guarantee the quality of the experiment, especially in recent years with the development of information technology, computer aided teaching has been widely introduced into the experimental teaching of Principle of Computer Composition [2-5]. But there are still some problems that need to be solved. Problems are mainly reflected as follows. More research focuses on the development of virtual experimental systems. There is no whole link to the experiment teaching and establishing corresponding auxiliary teaching platform, lacks research on how to apply the experimental auxiliary teaching platform. These can't provide guidance to practical applications. It is necessary to further explore the role of software in the learning of hardware curriculum knowledge.

Based on existing problems, we use the component technology in combination with the actual teaching requirements. The design and development of Principle of Computer Composition experiment teaching assistant platform based on MVC architecture. The platform supports the development of experimental teaching of computer composition in terms of curriculum information management, virtual experiment system, simulation program management and performance management. And the application method of the platform is discussed. The feasible schemes are proposed.

\section{Platform design}

\subsection{Platform functional design}

The experimental teaching assistant platform of Principle of Computer Composition is aiming at teachers and students. Teachers mainly provide the information sharing, job correction, performance management and other functions. Students mainly provide virtual experiment, simulation program, 
report upload and other functions. It provides the function of interaction between teachers and students. he platform functional structure design is shown in Fig.1.

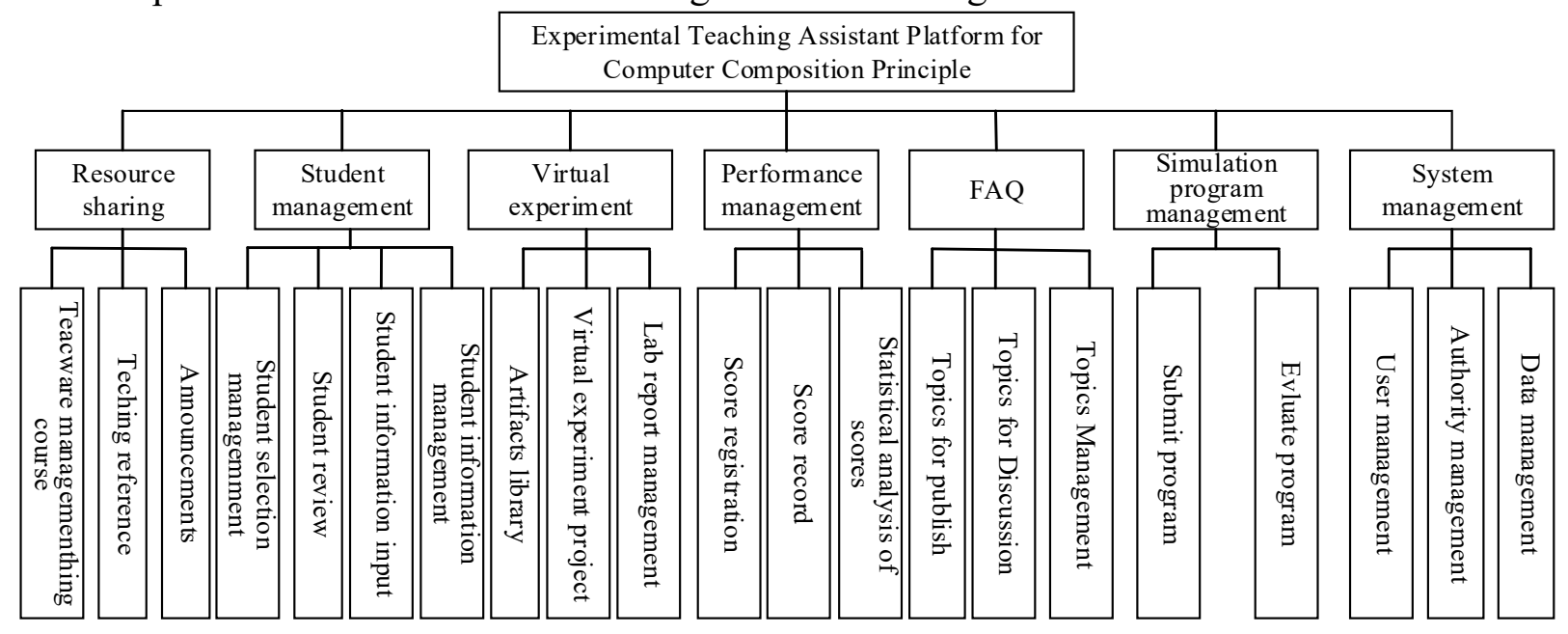

Fig. 1. Experiment teaching assistant platform functional structure

(i) Resource sharing

The module is mainly open to teachers and students to share information. It includes teaching courseware, experimental instruction, experiment instruction, software use manual, notice of course related notice, etc. Teachers can achieve the resource directory management, resource upload and input, resource maintenance, resource access management, resource download and browsing.

(ii) Student management

Teachers use the student management module to carry out the input, modification, deletion, batch data import and the function of student audit. Students use the student management module to implement the course selection. Only elective students can upload lab reports and statistics. It supports the standard Excel file data import in batch data import.

(iii) Virtual experiment management

The virtual experiment management is the biggest difference between the platform and other auxiliary teaching systems. It contains three sub-modules of the component library, virtual experiment, and implementation report management. The component library uses the component technology to provide some of the basic chips needed to complete the experiment. The virtual experiment project management mainly provides three experimental projects management which are the arithmetic logic operation unit, the static random memory reading and writing, and the simple model machine design. Students use the components in the component library to simulate the line operation by dragging and dropping the mouse to form functional components. The experiment report management realizes the upload, modification and the scoring function of experiment reports. Students must upload and modify lab reports within the time limit set by teachers. Results of the experiment report are as part of the grade management.

(iv) Simulation program management

Since the course is involved in more complex principles, many principles are difficult to understand, so the platform introduces software simulation technology into the teaching of Principle of Computer Composition. It has been added the simulation program management module. Students adopt the software simulation technology to write the simulation of the hardware working mechanism. It is submitted to the platform through the program submission function. The platform automatically determines the correctness of the program and gives the results.

(v) Performance management

The student achievement mainly consists of the attendance, experiment report, simulation program, and virtual experiment. The attendance and test reports are manually entered by teachers according to the actual situation. Results of simulation programs and virtual experiments are automatically generated by the system. Teachers can set the weight of each achievement and make 
statistical analysis of grades according to need, such as by item statistics. Results of the statistics are shown in tables or graphs and can export out Excel files.

\section{(vi) Q\&A}

The discussion module is mainly introduced to facilitate interaction between teachers and students. Students can choose to enter the topic by interest, conduct topic release, delete topics, and view topics. But students can only delete their own topics. Teachers can delete other people's topics or top up some topics.

(vii) System management

The system management consists of three sub-functions of user management, permission management and data management. The data management implements data backup and recovery, as well as the logging management capabilities. The user management mainly realizes the management of user information, such as the user name, password, role, etc. The permission management enables all columns and functions to be specified. It has automatic and autonomic increase access function.

\section{The platform architecture}

The Principle of Computer Composition experiment teaching auxiliary platform adopts the architecture shown in Fig. 2. It is divided into the data layer, the data access layer, the business logic processing layer, and the display layer, respectively corresponding to the three layers architecture of the MVC (Model View Controller). The data layer provides a variety of data to be processed by the platform. Date is stored in the form of database and file. The data access layer provides the interface to access data. Through the data access interface, the previous layer only needs to call these interfaces, the corresponding database operation can be completed for the data persistence operation; The business logic layer mainly implements the business logic processing of the platform, this layer accepts the request made by users and invokes the data access interface on request to obtain the data and returns the result data to the presentation layer. The display layer provides human-computer interaction to achieve the acceptance and results of user requests. To improve the reusability of the code, the platform provides reusable resources in the form of components, the display layer, business logic processing layer, and part of the data access layer are implemented by the component assembly, which simplifies the difficulty of the platform implementation and facilitates the later extension.

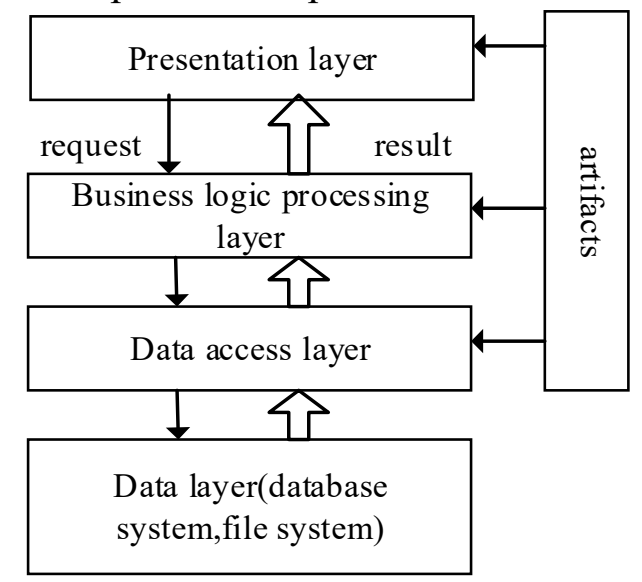

Fig.2. The platform architecture

\section{Application of platform}

In order to test the experimental auxiliary teaching platform, we have applied it in the computer application students of the 2014 grade in computer science department and achieved good results. In practice, we mainly pay attention to the following aspects.

(i) Materials of the lecture are all entered into the platform for students to view and download. In the experimental class, the traditional experimental box was used to record the students' attendance and the completion of the experiment and give the results in a quantitative way. Students fill out the experiment report online, the teacher corrects the experiment report and gives the result. 
(ii)After class, students are required to enter the platform, combine the experiment in the internal experiment box, conduct the virtual experiment, and give the normal results according to the number and quality of the virtual experiment project.

(iii) Students are required to use the software simulation technology to write the relevant working principles, and principles are submitted through the platform automatically, and the relevant results are generated.

(iv) Encourage students in accordance with the platform component design specification, write their own related components, more tests and complete the project, such as the complex model design experiment, the pipeline design experiment and the vector processor.

(v) To give full play to the function of platform answer discussion, the teacher participates in the discussion, actively guides the students to carry out the discussion on related issues, and strengthens the solution of the problem and the collision of new ideas.

\section{Conclusion}

It is using the means of informatization to carry out the Principle of Computer Composition experiment auxiliary teaching. It breaks the time and space constraints that existed in previous teaching and fully inspires the students' autonomous learning ability and creative thinking. The quality of curriculum teaching is improved effectively. At the same time, it also makes the examination of achievement more fair and reasonable. The next step is to extend the functionality of the platform, increase the subsystem of the question bank and the online test system and apply it into the theory course. The corresponding mobile app should be also developed to better play the role of auxiliary teaching.

\section{Acknowledgement}

This paper is supported by Principles of Computer Organization high-quality curriculum construction project of Chengdu University of Information Technology, and the construction stage research achievements of the fifth batch of characteristic specialties of the Ministry of Education Computer Science and Technology (TS2488).

\section{References}

[1] Q. Y. Zhao, J. He, and L Wei, Discussion on the teaching mode of Computer Organization and Architecture, Computer Knowledge and Technology, vol. 3, pp. 693-694, 2008.

[2] J. X. Yang, The exploration and practice of computer composition principle experiment network teaching, Laboratory Science, vol. 3, pp. 45-47, 2008

[3] W. F. Zhang, J. Xiao, and H. Y. Liu, Design and implementation of virtual experiment system for computer composition principle, Research and Exploration in Laboratory, vol. 5, pp. 62-66, 2014.

[4] H. Y. Zhang, Development of experimental simulation coursewares of principle of computer organization, Modern Computer, vol. 6, pp. 157-160, 2010.

[5] J. X. Wang, L. Y. Zhang, and Y. Sheng, Design and implementation of principles of computer organization virtual lab based on component, Journal of System Simulation, vol. 9, pp. 2469-2474, 2008. 